\title{
Basal Insulin Initiation and Maintenance in Adults with Type 2 Diabetes Mellitus in the United States
}

This article was published in the following Dove Press journal:

Diabetes, Metabolic Syndrome and Obesity: Targets and Therapy

\author{
Samaneh Kalirai' \\ Jasmina I Ivanova $\mathbb{D}^{2}$ \\ Magaly Perez-Nieves (iD) \\ Judith J Stephenson $\mathbb{D}^{3}$ \\ Irene Hadjiyianni $\mathbb{D}^{\prime}$ \\ Michael Grabner (D) $^{3}$ \\ Roy Daniel Pollom' \\ Caroline Geremakis ${ }^{3}$ \\ Beverly L Reed' \\ Lawrence Fisher (1D ${ }^{4}$ \\ 'Eli Lilly and Company, Indianapolis, IN, \\ USA; ${ }^{2}$ Analysis Group Inc., New York, \\ NY, USA; ${ }^{3}$ HealthCore Inc., Wilmington, \\ DE, USA; ${ }^{4}$ Department of Family and \\ Community Medicine, UC San Francisco, \\ San Francisco, CA, USA
}

Correspondence: Michael Grabner HealthCore, Inc., 123 Justison St, Suite 200, Wilmington, DE 1980I, USA Tel +l 302 230-2000

Email mgrabner@healthcore.com

\begin{abstract}
Objective: A survey of US adults with type 2 diabetes mellitus was conducted to better understand patients' insulin initiation experiences and treatment persistence behaviors.

Research Design and Methods: Participants were recruited from consumer panels and grouped by basal insulin treatment pattern: continuers (no gap of $\geq 7$ days within 6 months of initiation); interrupters (gap $\geq 7$ days, resumed treatment); discontinuers (stopped for $\geq 7$ days, not resumed). A quota of approximately 50 respondents per persistence category was set.

Results: A total of 154 respondents (52 continuers, 52 interrupters, 50 discontinuers) completed the survey. Mean age was 51.4 years; $51.9 \%$ male. Continuers were more likely to report their views being considered during initiation, and less likely to report a sense of failure. Concerns included insulin dependence (64.3\% agree/strongly agree), frequent blood glucose monitoring (55.2\%), costs/ability to pay (53.9\%), fears of or mistakes during self-injection $(52.6 \%)$, and weight gain (52.6\%). Continuers were motivated by benefits of insulin therapy; experienced or potential side effects were notable factors for interruption/discontinuation. Healthcare provider instruction was indicated as a reason for continuing, stopping, and restarting therapy.

Conclusion: Benefits of basal insulin therapy motivated continuers while side effects impacted interruption/discontinuation. Persistence on basal insulin is often influenced by provider actions. Earlier provider intervention upon signs of treatment discontinuation may promote persistence.

Keywords: type 2 diabetes mellitus, basal insulin, patient survey, medication persistence
\end{abstract}

\section{Plain Language Summary}

Many patients with type 2 diabetes mellitus require insulin treatment to control their blood sugar and prevent disease progression, but treatment initiation and persistence remain suboptimal, even when there is a clear need for insulin therapy.

Patient perspectives were collected from 154 respondents, representing insulin continuers, interrupters, and discontinuers. Persistence with insulin therapy varied with patient buy-in during the decision to start insulin. Benefits of basal insulin therapy motivated continuers while side effects were associated with interruption and discontinuation. Persuasion by providers was the most common reason for restarting insulin treatment. There are gaps between patient-reported concerns and physicians' perceptions of patient concerns.

The results suggest that patient buy-in when initiating insulin is likely to improve persistence, and earlier provider intervention, including explaining and addressing patients' concerns including potential side effects, may help reduce the amount of time patients are off their insulin therapy.

\section{Introduction}

Many patients with type 2 diabetes mellitus (T2DM) require insulin therapy to control their blood sugar and address the progressively deleterious effects of the disease. ${ }^{1-4}$ Treatment initiation, however, is often met with resistance, even when the need for 
insulin therapy is clear. ${ }^{5}$ Concerns persist despite the favorable track record of insulin use and considerable supporting clinical evidence and treatment guidelines. ${ }^{1-4,6,7}$ The perceptions of both patients and physicians often stymie treatment initiation and can prolong the time spent by untreated patients coping with hyperglycemia and poor glucose control. ${ }^{1,6,8-11}$

In addition to challenges with insulin initiation, poor medication persistence is also problematic in the management of T2DM and other chronic diseases. ${ }^{6,12,13}$ Low persistence rates negatively impact patients' long-term clinical outcomes and healthcare costs. ${ }^{14-16}$ Differences in how patients and primary care physicians (PCPs) perceive and take action regarding initiation and day-to-day maintenance of insulin therapy can negatively influence overall persistence. ${ }^{14,17-21}$ Knowledge about risk factors for poor persistence may assist physicians in identifying high-risk patients and tailoring interventions to help patients optimize treatment outcomes. ${ }^{22}$ Other important factors in supporting patients' transitions to insulin include assessing patients' feelings and experiences about insulin initiation and maintenance as well as identifying physicians' perceptions of patient reactions and concerns. ${ }^{23}$

In an earlier claims-based study, we examined persistence patterns with basal insulin in a large commercially insured population. During the first year after initiation, only $19.8 \%$ of patients continued insulin treatment regularly, $62.2 \%$ had $\geq 1$ interruption (defined as a gap of $\geq 30$ days), and $18.0 \%$ discontinued therapy. Most treatment interruptions and discontinuations occurred within the first three months after initiation; continuers had higher pharmacy costs but lower medical costs relative to interrupters and discontinuers. ${ }^{9}$ The study also sought to identify patient factors statistically associated with a higher likelihood of discontinuing basal insulin; among the contributing factors were younger age, female gender, diabetic foot complications, neurological disorders, and the occurrence of at least one endocrinologist visit or inpatient or emergency department visit prior to basal insulin initiation. Patients presenting with these characteristics may benefit from additional management/ risk assessments by their treating physicians.

In another prior study, we surveyed PCPs to examine their perceptions of patients' reactions and concerns around the initiation and maintenance of insulin therapy. The results showed that PCPs were often the ones recommending patients to begin insulin therapy. The study also suggested that better support from PCP offices incorporating education, outreach, titration algorithms, and better monitoring could be of value to patients. ${ }^{23}$

With the goal of adding an important dimension to the overall picture - the patient's perspective - the current analysis reports findings of a survey among T2DM patients in the United States (US) on their perceptions and experiences around the initiation and short-term use of basal insulin therapy and the self-reported reasons for different patterns of persistence over 6 months from initiation.

\section{Methods}

\section{Data Source and Study Population}

The data presented here are from the US portion of a multinational online survey, conducted between July and September 2015 and reported in detail elsewhere. ${ }^{24,25}$ Individuals identified from the Harris Panel (including its chronic illness sub-panel) and other third-party panels were included. The survey did not collect any personal identifying information, and the Western Institutional Review Board granted review exemption.

\section{Inclusion/Exclusion Criteria}

Respondents included in this analysis reported a diagnosis of T2DM and were $\geq 18$ years old. Inclusion also required initiation of basal insulin analog therapy - insulin glargine, detemir or isophane - during the immediately preceding 3-24 months, and being insulin-naïve prior to starting treatment. Respondents who interrupted or stopped using basal insulin after the first 6 months following insulin initiation were not included in the analysis. Also excluded were respondents who were pregnant or breastfeeding at the time of or after insulin initiation.

\section{Persistence Groups}

Respondents were categorized into three persistence groups based on their self-reported treatment patterns. Respondents who reported no gaps of $\geq 7$ days in basal insulin treatment between initiation and the time of the survey were classified as continuers. Those who had a gap in their basal insulin usage for $\geq 7$ days within the first 6 months after initiation but restarted basal insulin use prior to the survey were categorized as interrupters. Those who stopped using basal insulin for $\geq 7$ days within the first 6 months after initiation and had not restarted insulin therapy by the time of the survey were classified as discontinuers. 


\section{Survey Methodology and Statistical Analysis}

This paper describes US-specific data collected as part of a cross-sectional online survey administered to a total of 942 respondents from the US and 6 other countries (Germany, France, Japan, Spain, UK, and Brazil) between July and September 2015. The overall study employed an approximate target quota of 50 respondents per persistence category per country to allow for exploratory analysis by country, and enrollment ceased upon attaining the approximate target or when the target could not be achieved after further time and recruitment effort. Among the topics covered in the survey were respondent demographic and socioeconomic characteristics, disease and treatment history, basal insulin initiation experience, experiences while taking basal insulin, and reasons for different patterns of persistence while on insulin therapy (ie, continue on therapy, early discontinuation, early interruption). The survey was developed based on previous findings from semistructured qualitative interviews of adults with T2DM from the same set of countries.

Questions about feelings when considering insulin were assessed on a 1-5 scale (1="Not at all", 2="Slightly", 3="Somewhat", 4="Very", 5="Fully"). Concerns before starting insulin were assessed on a 1-5 level of agreement scale (1="Strongly disagree", 2="Disagree", 3="Neither agree nor disagree", 4="Agree", 5="Strongly agree"). Challenges during the first week of insulin use were assessed on a 4 point scale (1="Not at all difficult", 2="Somewhat difficult", $3=$ ="Difficult", 4="Very difficult"). Self-reported reasons for different patterns of persistence were based on selecting specific items from a pre-specified list of potential reasons or entering information for other not-specified reasons.

Means and standard deviations were used to summarize continuous measures and proportions were reported for categorical variables. Pairwise comparisons between persistence groups were made using t-tests for continuous variables and chi-square or Fisher's exact tests of significance for categorical variables. Statistical significance was defined as $\mathrm{p}<0.05$.

\section{Results}

\section{Respondent Characteristics}

The US study sample consisted of a total of 154 respondents - 52 continuers (33.8\%), 52 interrupters (33.8\%), and 50 discontinuers (32.4\%). Mean age was 51.4 years; $51.9 \%$ of respondents were male. On average, continuers were significantly older than interrupters and discontinuers (mean age: 63.8 vs $43.2(\mathrm{p}<0.01)$ and $47.2(\mathrm{p}<0.01)$ years, respectively); discontinuers had less prior use of antidiabetic medications than continuers or interrupters $(60.0 \%$ vs $82.7 \%(\mathrm{p}=0.01)$ and $86.5 \%(\mathrm{p}<0.01)$, respectively $)$. Other characteristics for each group are shown in Table 1.

\section{Insulin Use Experience Across Persistence Groups}

In this survey, $90.4 \%$ of continuers reported that their views were somewhat or very/fully considered in the decision to start insulin, compared to $76.9 \%$ of interrupters and $80.0 \%$ of discontinuers. When considering insulin therapy, a significantly lower proportion $(11.5 \%)$ of continuers believed that insulin was not necessary compared with interrupters $(44.2 \%, \mathrm{p}<0.01)$ and discontinuers $(34.0 \%, \mathrm{p}<0.01)$. In addition, continuers (40.4\%) were significantly less likely to report a sense of failure than interrupters $(63.5 \%, \mathrm{p}=0.02)$, as shown in Table 2 .

The most common concerns at the time of insulin initiation were about insulin dependence $(64.3 \%$ agree or strongly agree), a need to monitor blood glucose more often (55.2\%), ability to pay for insulin therapy (53.9\%), fear of self-injection (52.6\%), fear of making mistakes during self-injection $(52.6 \%)$, and worry about potential weight gain $(52.6 \%)$, as shown in Figure 1.

After the first week of using insulin, significantly lower proportions of continuers reported that they agreed/ strongly agreed with having specific concerns related to the fear of making mistakes during self-injection and concerns about potentially frequent hypoglycemia. For example, before starting insulin, $32.7 \%$ of continuers agreed or strongly agreed that they feared self-injection compared with $69.2 \%$ of interrupters $(\mathrm{p}<0.01)$ and $56.0 \%$ of discontinuers $(p=0.02)$, as shown in Figure 2. Significantly lower proportions $(0-12 \%)$ of continuers reported experiencing specific challenges (related to injecting insulin, more frequent blood glucose monitoring, titration, proper storage, remembering to take insulin, emotions related to needing insulin) during the first week of insulin initiation than interrupters and discontinuers ( $8 \%$ to $48 \%$ ). For example, $3.8 \%$ of continuers expressed challenges with injecting insulin compared with $28.8 \%$ of interrupters $(p<0.01)$ and $20.0 \%$ of discontinuers $(p=0.01)$. Only $1.9 \%$ of continuers reported that they considered remembering to inject insulin regularly difficult or very difficult compared with $48.1 \%$ of interrupters $(p<0.01)$ and $26.0 \%$ of discontinuers $(\mathrm{p}<0.01)$, as shown in Appendix Table 1. 
Table I Baseline Patient Characteristics

\begin{tabular}{|c|c|c|c|c|c|c|c|c|c|c|c|}
\hline \multirow{4}{*}{$\begin{array}{l} \\
\text { Age } \\
\text { Mean (SD) }\end{array}$} & \multirow{2}{*}{\multicolumn{2}{|c|}{$\begin{array}{l}\text { Overall } \\
(n=154)\end{array}$}} & \multirow{2}{*}{\multicolumn{2}{|c|}{$\begin{array}{l}\text { Continuers } \\
{[A]} \\
(n=52)\end{array}$}} & \multirow{2}{*}{\multicolumn{2}{|c|}{$\begin{array}{l}\text { Interrupters } \\
{[B]} \\
(n=52)\end{array}$}} & \multirow{2}{*}{\multicolumn{2}{|c|}{$\begin{array}{l}\text { Discontinuers } \\
{[C]} \\
(n=50)\end{array}$}} & \multirow{4}{*}{$\begin{array}{l}\text { p-value } \\
\text { [A vs B] } \\
<0.01\end{array}$} & \multirow{4}{*}{$\begin{array}{l}\text { p-value } \\
\text { [A vs C] } \\
<0.01\end{array}$} & \multirow{4}{*}{$\begin{array}{l}\text { p-value } \\
{[B \text { vs C }]} \\
0.16\end{array}$} \\
\hline & & & & & & & & & & & \\
\hline & \multirow{2}{*}{51.} & \multirow[b]{2}{*}{$(16.4)$} & \multirow{2}{*}{63.8} & \multirow[b]{2}{*}{ (12.5) } & \multirow[b]{2}{*}{43.2} & \multirow[b]{2}{*}{$(14.0)$} & \multirow[b]{2}{*}{47.2} & \multirow[b]{2}{*}{ (14.8) } & & & \\
\hline & & & & & & & & & & & \\
\hline Male, $n(\%)$ & 80 & $(51.9 \%)$ & 28 & $(53.8 \%)$ & 32 & $(61.5 \%)$ & 20 & $(40.0 \%)$ & 0.43 & 0.16 & 0.03 \\
\hline Race/ethnicity, n (\%)* & & & & & & & & & & & \\
\hline White & 124 & $(80.5 \%)$ & 47 & $(90.4 \%)$ & 37 & $(71.2 \%)$ & 40 & $(80.0 \%)$ & 0.01 & 0.14 & 0.30 \\
\hline Black or African American & 23 & $(14.9 \%)$ & 6 & (II.5\%) & 10 & $(19.2 \%)$ & 7 & $(14.0 \%)$ & 0.28 & 0.71 & 0.48 \\
\hline Hispanic & 9 & $(5.8 \%)$ & 1 & $(1.9 \%)$ & 3 & $(5.8 \%)$ & 5 & $(10.0 \%)$ & 0.31 & 0.08 & 0.43 \\
\hline American Indian or Alaska Native & 3 & $(1.9 \%)$ & 0 & $(0.0 \%)$ & 0 & $(0.0 \%)$ & 3 & $(6.0 \%)$ & - & 0.07 & 0.07 \\
\hline Asian & 5 & $(3.2 \%)$ & 0 & $(0.0 \%)$ & 4 & $(7.7 \%)$ & 1 & $(2.0 \%)$ & 0.04 & 0.31 & 0.18 \\
\hline Education level, $\mathbf{n}(\%)$ & & & & & & & & & 0.01 & 0.12 & 0.61 \\
\hline Less than high school & 1 & $(0.6 \%)$ & 0 & $(0.0 \%)$ & I & $(1.9 \%)$ & 0 & $(0.0 \%)$ & & & \\
\hline High school or equivalent & 25 & $(16.2 \%)$ & 13 & $(25.0 \%)$ & 5 & $(9.6 \%)$ & 7 & $(14.0 \%)$ & & & \\
\hline Some college & 44 & $(28.6 \%)$ & 17 & $(32.7 \%)$ & 12 & $(23.1 \%)$ & 15 & $(30.0 \%)$ & & & \\
\hline Associate's degree or equivalent & 20 & $(13.0 \%)$ & 4 & $(7.7 \%)$ & 7 & $(13.5 \%)$ & 9 & $(\mid 8.0 \%)$ & & & \\
\hline College degree & 64 & $(41.6 \%)$ & 18 & $(34.6 \%)$ & 27 & $(51.9 \%)$ & 19 & $(38.0 \%)$ & & & \\
\hline Bachelor's degree or equivalent & 47 & $(30.5 \%)$ & 9 & $(17.3 \%)$ & 23 & $(44.2 \%)$ & 15 & $(30.0 \%)$ & & & \\
\hline Graduate degree & 17 & $(11.0 \%)$ & 9 & $(17.3 \%)$ & 4 & $(7.7 \%)$ & 4 & $(8.0 \%)$ & & & \\
\hline Employment status, n (\%) & & & & & & & & & $<0.01$ & $<0.01$ & 0.03 \\
\hline Working full-time & 64 & $(41.6 \%)$ & II & $(21.2 \%)$ & 32 & $(61.5 \%)$ & 21 & $(42.0 \%)$ & & & \\
\hline Working part-time & 15 & $(9.7 \%)$ & 2 & $(3.8 \%)$ & 6 & (11.5\%) & 7 & $(14.0 \%)$ & & & \\
\hline Not employed & 5 & $(3.2 \%)$ & 1 & $(1.9 \%)$ & 2 & $(3.8 \%)$ & 2 & $(4.0 \%)$ & & & \\
\hline Disabled & 14 & $(9.1 \%)$ & 2 & $(3.8 \%)$ & 2 & $(3.8 \%)$ & 10 & $(20.0 \%)$ & & & \\
\hline Retired & 41 & $(26.6 \%)$ & 29 & $(55.8 \%)$ & 4 & $(7.7 \%)$ & 8 & $(16.0 \%)$ & & & \\
\hline Student & 5 & $(3.2 \%)$ & 0 & $(0.0 \%)$ & 5 & $(9.6 \%)$ & 0 & $(0.0 \%)$ & & & \\
\hline Homemaker & 10 & $(6.5 \%)$ & 7 & $(13.5 \%)$ & I & $(1.9 \%)$ & 2 & $(4.0 \%)$ & & & \\
\hline Living with a spouse/partner, $n$ (\%) & 105 & $(68.2 \%)$ & 35 & $(67.3 \%)$ & 37 & $(71.2 \%)$ & 33 & $(66.0 \%)$ & 0.67 & 0.89 & 0.58 \\
\hline $\begin{array}{l}\text { Health insurance coverage when } \\
\text { initiating basal insulin, } n(\%)\end{array}$ & & & & & & & & & & & \\
\hline Medical care & 137 & $(89.0 \%)$ & 51 & (98.1\%) & 44 & $(84.6 \%)$ & 42 & $(84.0 \%)$ & 0.02 & 0.01 & 0.93 \\
\hline Prescription drugs & 138 & $(89.6 \%)$ & 50 & $(96.2 \%)$ & 47 & $(90.4 \%)$ & 41 & $(82.0 \%)$ & 0.24 & 0.02 & 0.22 \\
\hline Years since first T2DM diagnosis & & & & & & & & & & & \\
\hline Mean (SD) & 8.6 & $(9.3)$ & 9.9 & (7.0) & 8.1 & $(11.2)$ & 7.8 & (9.5) & 0.32 & 0.21 & 0.89 \\
\hline Median [IQR] & 6.0 & {$[3.0-12.0]$} & 10.0 & {$[4.0-14.5]$} & 6.0 & {$[3.0-10.0]$} & 4.5 & {$[2.0-10.0]$} & & & \\
\hline $\begin{array}{l}\text { Mode of delivery of basal insulin } \\
\text { initiated, } n(\%)\end{array}$ & & & & & & & & & 0.57 & 0.15 & 0.50 \\
\hline Pen (prefilled/disposable) & 97 & $(63.0 \%)$ & 37 & (71.2\%) & 32 & $(61.5 \%)$ & 28 & $(56.0 \%)$ & & & \\
\hline Pen (reusable) \& cartridge & 20 & $(\mid 3.0 \%)$ & 4 & $(7.7 \%)$ & 6 & (II.5\%) & 10 & $(20.0 \%)$ & & & \\
\hline Vial and syringe & 37 & $(24.0 \%)$ & II & $(21.2 \%)$ & 14 & $(26.9 \%)$ & 12 & $(24.0 \%)$ & & & \\
\hline $\begin{array}{l}\text { Prior use of antidiabetic medications } \\
\text { to treat T2DM, } n(\%)\end{array}$ & & & & & & & & & & & \\
\hline Any prior use of antidiabetic medication & 118 & $(76.6 \%)$ & 43 & $(82.7 \%)$ & 45 & $(86.5 \%)$ & 30 & $(60.0 \%)$ & 0.59 & 0.01 & $<0.01$ \\
\hline Oral antidiabetics & 105 & $(68.2 \%)$ & $4 I$ & $(78.8 \%)$ & 37 & $(71.2 \%)$ & 27 & $(54.0 \%)$ & 0.37 & $<0.01$ & 0.07 \\
\hline Injectables other than insulin & 30 & $(19.5 \%)$ & 8 & $(15.4 \%)$ & 15 & $(28.8 \%)$ & 7 & $(14.0 \%)$ & 0.10 & 0.84 & 0.07 \\
\hline
\end{tabular}

Notes: Continuers had no gaps of $\geq 7$ days in basal insulin treatment. Interrupters stopped basal insulin for $\geq 7$ days within the first 6 months after initiation and since restarted basal insulin. Discontinuers stopped using basal insulin for $\geq 7$ days within the first 6 months after initiation and had not restarted basal insulin by the time of the survey. *One respondent declined to answer this question. Categories not listed in the table had 0 respondents ("Native Hawaiian or other Pacific Islander", "Other"). $\mathrm{P}$-values were calculated using t-tests for continuous variables and chi-square tests or Fisher's exact tests for categorical variables. $\mathrm{P}<0.05$ was considered statistically significant. 
Table 2 Perceptions About Insulin Use and Experience Across Persistence Groups

\begin{tabular}{|c|c|c|c|c|c|c|c|c|c|c|c|}
\hline \multirow[b]{3}{*}{$\begin{array}{l}\text { Respondent's motivations for starting insulin, n (\%) } \\
\text { responding "Yes" }\end{array}$} & \multicolumn{2}{|c|}{ Overall } & \multicolumn{2}{|c|}{$\begin{array}{l}\text { Continuers } \\
\text { [A] }\end{array}$} & \multicolumn{2}{|c|}{$\begin{array}{l}\text { Interrupters } \\
\text { [B] }\end{array}$} & \multicolumn{2}{|c|}{$\begin{array}{l}\text { Discontinuers } \\
{[C]}\end{array}$} & \multirow[t]{2}{*}{$\begin{array}{l}\text { P-value } \\
{[A \text { vs B] }}\end{array}$} & \multirow[t]{2}{*}{$\begin{array}{l}\text { p-value } \\
\text { [A vs C] }\end{array}$} & \multirow[t]{2}{*}{$\begin{array}{l}\text { p-value } \\
{[B \text { vs C }]}\end{array}$} \\
\hline & \multicolumn{2}{|c|}{$(n=154)$} & \multicolumn{2}{|c|}{$(n=52)$} & \multicolumn{2}{|c|}{$(n=52)$} & \multicolumn{2}{|c|}{$(n=50)$} & & & \\
\hline & & & & & & & & & & & \\
\hline Encouragement from physician/healthcare provider & 101 & $(65.6 \%)$ & 40 & $(76.9 \%)$ & 30 & $(57.7 \%)$ & 31 & $(62.0 \%)$ & 0.04 & 0.10 & 0.66 \\
\hline Improved glycemic control & 92 & $(59.7 \%)$ & 26 & $(50.0 \%)$ & 36 & $(69.2 \%)$ & 30 & $(60.0 \%)$ & $<0.05$ & 0.31 & 0.33 \\
\hline $\begin{array}{l}\text { Concern about developing other complications of } \\
\text { diabetes }\end{array}$ & 42 & $(27.3 \%)$ & 16 & $(30.8 \%)$ & 13 & $(25.0 \%)$ & 13 & $(26.0 \%)$ & 0.51 & 0.59 & 0.91 \\
\hline Preference for injections over pills & 10 & $(6.5 \%)$ & I & $(1.9 \%)$ & 4 & $(7.7 \%)$ & 5 & $(10.0 \%)$ & 0.17 & 0.08 & 0.68 \\
\hline Inability to tolerate other antidiabetic medications & 4 & $(2.6 \%)$ & 2 & $(3.8 \%)$ & 1 & $(1.9 \%)$ & $\mathrm{I}$ & $(2.0 \%)$ & 0.56 & 0.58 & 0.98 \\
\hline Other & 3 & $(1.9 \%)$ & 2 & $(3.8 \%)$ & 0 & $(0.0 \%)$ & 1 & $(2.0 \%)$ & 0.15 & 0.58 & 0.31 \\
\hline $\begin{array}{l}\text { Degree to which respondent felt views were } \\
\text { considered, } n(\%)\end{array}$ & & & & & & & & & 0.06 & 0.33 & 0.30 \\
\hline Not at all/slightly & 27 & $(17.5 \%)$ & 5 & $(9.6 \%)$ & 12 & $(23.1 \%)$ & 10 & $(20.0 \%)$ & & & \\
\hline Somewhat & 38 & $(24.7 \%)$ & 16 & $(30.8 \%)$ & 8 & $(15.4 \%)$ & 14 & $(28.0 \%)$ & & & \\
\hline Very/fully & 89 & $(57.8 \%)$ & 31 & $(59.6 \%)$ & 32 & $(61.5 \%)$ & 26 & $(52.0 \%)$ & & & \\
\hline Converted to 0-100, mean (SD) & 63.5 & $(27.3)$ & 68.8 & (26.6) & 61.1 & (28.6) & 60.5 & $(26.3)$ & 0.03 & 0.24 & 0.54 \\
\hline $\begin{array}{l}\text { Feelings when considering insulin, } \mathbf{n}(\%) \text { who agree/ } \\
\text { strongly agree }\end{array}$ & & & & & & & & & & & \\
\hline Reassurance that insulin would help manage diabetes & 122 & $(79.2 \%)$ & 41 & $(78.8 \%)$ & 45 & $(86.5 \%)$ & 36 & $(72.0 \%)$ & 0.30 & 0.42 & 0.07 \\
\hline Fear of developing other complications of diabetes & 115 & (74.7\%) & 40 & $(76.9 \%)$ & 43 & $(82.7 \%)$ & 32 & $(64.0 \%)$ & 0.46 & 0.15 & 0.03 \\
\hline Feeling that insulin indicated that diabetes was worsening & 108 & $(70.1 \%)$ & 35 & $(67.3 \%)$ & 39 & $(75.0 \%)$ & 34 & $(68.0 \%)$ & 0.39 & 0.94 & 0.43 \\
\hline Sense of failure & 72 & $(46.8 \%)$ & 21 & $(40.4 \%)$ & 33 & $(63.5 \%)$ & 18 & $(36.0 \%)$ & 0.02 & 0.65 & $<0.01$ \\
\hline Belief that insulin was not necessary & 46 & $(29.9 \%)$ & 6 & (11.5\%) & 23 & $(44.2 \%)$ & 17 & $(34.0 \%)$ & $<0.01$ & $<0.01$ & 0.29 \\
\hline $\begin{array}{l}\text { Concerns before starting insulin, } n(\%) \text { who agree/ } \\
\text { strongly agree }\end{array}$ & & & & & & & & & & & \\
\hline Concern about becoming insulin-dependent & 99 & $(64.3 \%)$ & 25 & $(48.1 \%)$ & 38 & $(73.1 \%)$ & 36 & $(72.0 \%)$ & $<0.01$ & 0.01 & 0.90 \\
\hline $\begin{array}{l}\text { Concern that he/she would need to monitor blood glucose } \\
\text { more often }\end{array}$ & 85 & $(55.2 \%)$ & 21 & $(40.4 \%)$ & 38 & $(73.1 \%)$ & 26 & $(52.0 \%)$ & $<0.01$ & 0.24 & 0.03 \\
\hline Concern about ability to pay for insulin therapy & 83 & $(53.9 \%)$ & 22 & $(42.3 \%)$ & 38 & $(73.1 \%)$ & 23 & $(46.0 \%)$ & $<0.01$ & 0.71 & $<0.01$ \\
\hline Fear of self-injection & 81 & $(52.6 \%)$ & 17 & $(32.7 \%)$ & 36 & $(69.2 \%)$ & 28 & $(56.0 \%)$ & $<0.01$ & 0.02 & 0.17 \\
\hline Fear of making mistakes during self-injection & 81 & $(52.6 \%)$ & 16 & $(30.8 \%)$ & 38 & $(73.1 \%)$ & 27 & $(54.0 \%)$ & $<0.01$ & 0.02 & $<0.05$ \\
\hline Worry about potential weight gain & 81 & $(52.6 \%)$ & 20 & $(38.5 \%)$ & 36 & $(69.2 \%)$ & 25 & $(50.0 \%)$ & $<0.01$ & 0.24 & $<0.05$ \\
\hline Concern about carrying insulin around & 72 & $(46.8 \%)$ & 20 & $(38.5 \%)$ & 32 & $(61.5 \%)$ & 20 & $(40.0 \%)$ & 0.02 & 0.87 & 0.03 \\
\hline $\begin{array}{l}\text { Concern that he/she would need to visit physician/nurse } \\
\text { more often }\end{array}$ & 71 & $(46.1 \%)$ & 16 & $(30.8 \%)$ & 34 & $(65.4 \%)$ & 21 & $(42.0 \%)$ & $<0.01$ & 0.24 & 0.02 \\
\hline Worry about injecting insulin in front of other people & 69 & $(44.8 \%)$ & 15 & $(28.8 \%)$ & 31 & $(59.6 \%)$ & 23 & $(46.0 \%)$ & $<0.01$ & 0.07 & 0.17 \\
\hline Worry about proper insulin storage & 64 & $(41.6 \%)$ & 13 & $(25.0 \%)$ & 31 & $(59.6 \%)$ & 20 & $(40.0 \%)$ & $<0.01$ & 0.11 & $<0.05$ \\
\hline $\begin{array}{l}\text { Worry that regular insulin use would interfere with daily } \\
\text { activities }\end{array}$ & 64 & $(41.6 \%)$ & 12 & $(23.1 \%)$ & 32 & $(61.5 \%)$ & 20 & $(40.0 \%)$ & $<0.01$ & 0.07 & 0.03 \\
\hline Worry that insulin would not provide glycemic control & 63 & $(40.9 \%)$ & 12 & $(23.1 \%)$ & 28 & $(53.8 \%)$ & 23 & $(46.0 \%)$ & $<0.01$ & 0.01 & 0.43 \\
\hline Concern about potentially frequent hypoglycemia & 60 & $(39.0 \%)$ & 9 & (17.3\%) & 27 & (51.9\%) & 24 & (48.0\%) & $<0.01$ & $<0.01$ & 0.69 \\
\hline Worry that scarring or bruising would result from injections & 55 & $(35.7 \%)$ & 9 & $(17.3 \%)$ & 29 & $(55.8 \%)$ & 17 & $(34.0 \%)$ & $<0.01$ & 0.05 & 0.03 \\
\hline
\end{tabular}

Notes: Continuers had no gaps of $\geq 7$ days in basal insulin treatment. Interrupters stopped basal insulin for $\geq 7$ days within the first 6 months after initiation and since restarted basal insulin. Discontinuers stopped using basal insulin for $\geq 7$ days within the first 6 months after initiation and had not restarted basal insulin by the time of the survey. P-values were calculated using t-tests for continuous variables and chi-square tests or Fisher's exact tests for categorical variables. P $<0.05$ was considered statistically significant. Feelings when considering insulin were assessed on a I-5 scale (I="Not at all", 2="Slightly", 3="Somewhat", 4="Very", 5="Fully"). Concerns before starting insulin were assessed on a $\mathrm{I}-5$ level of agreement scale (I="Strongly disagree", 2="Disagree", 3="Neither agree nor disagree", 4="Agree", 5="Strongly agree").

\section{Self-Reported Reasons for Persistence Behaviors}

Patients were asked about why they started and continued taking basal insulin; if they stopped, why they stopped or interrupted; and if they restarted, why they did so.

\section{Continuers}

The benefits of basal insulin therapy appeared to motivate continuers. The most common reasons reported by continuers for remaining on basal insulin therapy were improved glycemic control (78.8\%), the belief that insulin 


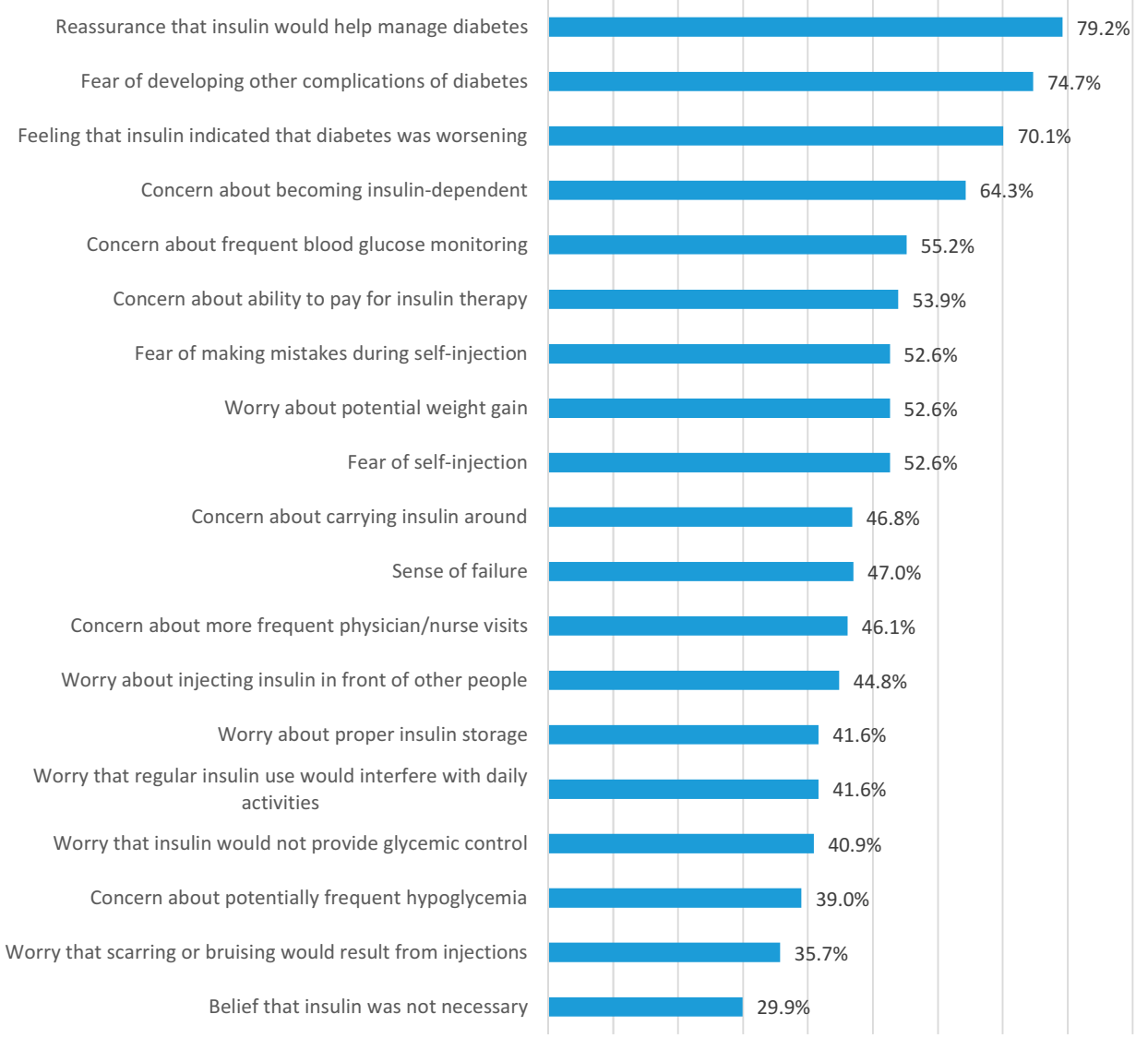

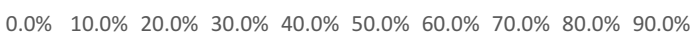

Figure I Patients' feelings, worries, and concerns when initiating basal insulin (combined across continuers, interrupters, discontinuers; $\mathrm{N}=\mathbf{1 5 4}$ ). Numbers reflect the percentage of patients who agree/strongly agree with a particular feeling, worry, or concern.

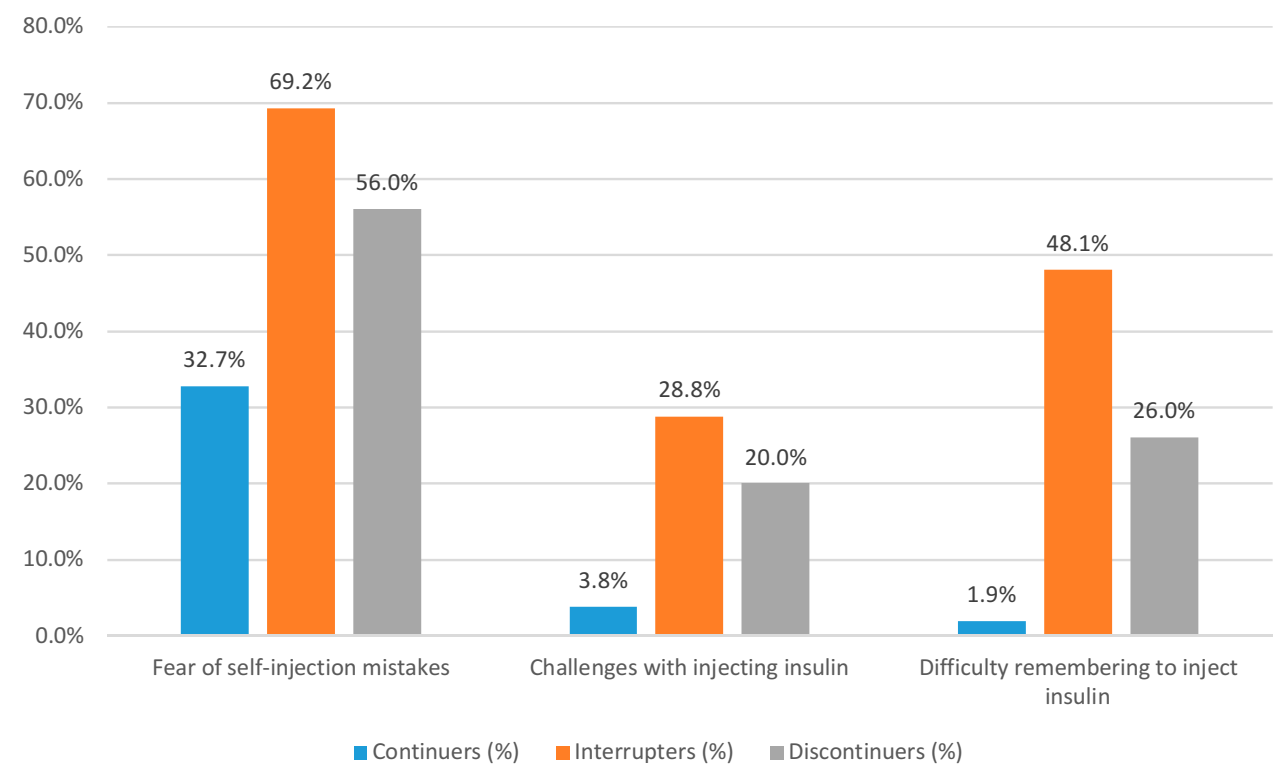

Figure 2 Insulin use experience after first week of initiation. All differences (continuers vs. interrupters or vs. discontinuers) are statistically significant at $p<0.05$. 
was best for reducing the risk of complications (63.5\%), instructions by a healthcare practitioner (HCP) to continue therapy (53.8\%), and improved physical feeling (44.2\%), as shown in Table 3.

\section{Interrupters}

Side effects, either experienced or potential, were most often noted as reasons for treatment interruptions. The most common reasons contributing to treatment interruption were weight gain (34.6\%), fear of potential side effects of insulin use (30.8\%), hypoglycemia $(23.1 \%)$, and the inconvenience of using insulin while traveling, at work, or at home (23.1\%). Assessing whether diabetes could be managed without insulin (21.2\%), instructions by HCP to stop (21.2\%), and the cost of insulin therapy (19.2\%) were also important reasons for interruption. The most common factors contributing to restarting basal insulin therapy among interrupters were persuasion by HCP $(69.2 \%)$ and persuasion by friends/family (36.5\%).

\section{Discontinuers}

Among respondents who discontinued, the most common contributory reasons were instructions by HCP to stop (46.0\%), weight gain $(30.0 \%)$, hypoglycemia $(24.0 \%)$, dislike/fear of needles $(20.0 \%)$, preference for oral antidiabetic medications $(20.0 \%)$, and the cost of insulin therapy $(20.0 \%)$. Persuasion by the HCP was the most commonly reported reason $(54.0 \%)$ contributing to insulin restarts among discontinuers. Even though side effects (experienced or potential) were often noted as reasons for interruption or discontinuation, rates of weight gain and hypoglycemia were similar between continuers and discontinuers (weight gain: $28.8 \%$ and $36.0 \%$; hypoglycemia: $26.9 \%$ and $34.0 \%$ ), although rates of hypoglycemia were higher among interrupters (weight gain: $42.3 \%$; hypoglycemia: $48.1 \%$ ) compared with continuers.

\section{Discussion}

Current guidelines for the management of T2DM recommend a stepwise approach, with the incorporation of insulin into the treatment regimen when deemed appropriate and likely to improve outcomes. ${ }^{2,7,26}$ The available evidence, however, consistently indicates lack of persistence to insulin by a significant percentage of patients. ${ }^{3}$ It is important to consider different perspectives when evaluating this issue. For example, results from our patient survey showed that therapy continuers were more likely to report that their views were somewhat or very/fully considered in the decision to start insulin. Such accord between providers and patients around the initiation of insulin may
Table 3 Self-Reported Reasons for Different Persistence Patterns

\begin{tabular}{|l|l|l|}
\hline \multicolumn{2}{|l|}{} & \multicolumn{2}{l|}{$\begin{array}{l}\text { Total } \\
\text { N = 154 }\end{array}$} \\
\hline Continuation (n=52) & & \\
\hline Motivations for continuation of basal insulin, & & \\
$\mathbf{n}(\%)$ & 4 I & $78.8 \%$ \\
Improved glycemic control & 33 & $63.5 \%$ \\
Belief that insulin is best for reducing risk of & & \\
complications of diabetes & 28 & $53.8 \%$ \\
Instruction by physician/healthcare provider to & & \\
continue & 23 & $44.2 \%$ \\
Improved physical feeling & 13 & $25.0 \%$ \\
Improved emotional wellbeing & 12 & $23.1 \%$ \\
Convenience of insulin relative to other diabetes & & \\
treatments & \\
\hline
\end{tabular}

Interruption $(\mathbf{n}=52)$

\begin{tabular}{|l|l|l|}
\hline More than one interruption, $\mathbf{n}(\%)$ & 40 & $76.9 \%$ \\
\hline Factors contributing to interruption, $\mathbf{n}(\%)$ & & \\
Weight gain & 18 & $34.6 \%$ \\
Fear of potential side effects of insulin use & 16 & $30.8 \%$ \\
Hypoglycemia & 12 & $23.1 \%$ \\
Inconvenience of using insulin & 12 & $23.1 \%$ \\
Instruction by physician/healthcare provider to stop & 11 & $21.2 \%$ \\
Assessing whether diabetes could be managed without & 11 & $21.2 \%$ \\
insulin & & \\
Cost of insulin therapy & 10 & $19.2 \%$ \\
Pain from injections & 9 & $17.3 \%$ \\
Preference for oral antidiabetic medications & 8 & $15.4 \%$ \\
Dislike/fear of needles & 7 & $13.5 \%$ \\
Insufficient glycemic control with insulin & 6 & $11.5 \%$ \\
Other & 2 & $3.8 \%$ \\
\hline Factors contributing to restarting basal insulin, & & \\
n (\%) & & \\
Persuasion by physician/healthcare provider to restart & 36 & $69.2 \%$ \\
Persuasion by friends/family to restart & 19 & $36.5 \%$ \\
Insufficient glycemic control without insulin & 18 & $34.6 \%$ \\
Resolution of the issue that led to interruption & 8 & $15.4 \%$ \\
Other & 2 & $3.8 \%$ \\
\hline
\end{tabular}

Discontinuation $(n=50)$

Factors contributing to discontinuation, $\mathbf{n}(\%)$ Instruction by physician/healthcare provider to stop

Weight gain

Hypoglycemia

Dislike/fear of needles

Preference for oral antidiabetic medications

Cost of insulin therapy

Insufficient glycemic control with insulin

Sense that diabetes could be managed without insulin

Pain from injections 
Table 3 (Continued).

\begin{tabular}{|l|l|l|}
\hline & \multicolumn{2}{|l|}{ Total } \\
\multicolumn{2}{|l|}{ N = 154 } \\
\hline Inconvenience of using insulin & 5 & $10.0 \%$ \\
Fear of potential side effects of insulin use & 3 & $6.0 \%$ \\
Perception of insulin use by others & 1 & $2.0 \%$ \\
Other & 6 & $12.0 \%$ \\
\hline Factors that could contribute to restarting & & \\
basal insulin, n (\%) & & \\
Persuasion by physician/healthcare provider to restart & 27 & $54.0 \%$ \\
Insufficient glycemic control without insulin & 22 & $44.0 \%$ \\
Persuasion by friends/family to restart & 12 & $24.0 \%$ \\
Nothing & 6 & $12.0 \%$ \\
Other & 2 & $4.0 \%$ \\
\hline
\end{tabular}

Notes: Continuers had no gaps of $\geq 7$ days in basal insulin treatment. Interrupters stopped basal insulin for $\geq 7$ days within the first 6 months after initiation and since restarted basal insulin. Discontinuers stopped using basal insulin for $\geq 7$ days within the first 6 months after initiation and had not restarted basal insulin by the time of the survey.

provide a foundation for better persistence later on. ${ }^{27-34}$ This process may also endow continuers with a sense of responsibility to better self-manage, find solutions for their own care, and to report fewer concerns and specific challenges after initiation. ${ }^{35-37}$

Experienced or potential weight gain and hypoglycemia are among the top reported reasons to interrupt or discontinue treatment. Better management of these side effects, for example through closer dietary management (for weight gain) and guidance on self-titration (for hypoglycemia), may help to alleviate respondent concerns. ${ }^{35-37}$ The most common reason respondents cited for restarting insulin treatment was persuasion by their provider, further highlighting the importance of the patient-provider relationship. The patient survey findings also align with evidence from our claims-based study ${ }^{9}$ in terms of patient factors associated with discontinuation; for example, in both data sets interrupters/discontinuers tended to be younger than continuers.

Considering providers, our earlier PCP survey ${ }^{23}$ collected information on patients' concerns about basal insulin initiation from the PCP perspective. These can be contrasted to the findings of the patient survey (with the limitation that the PCPs were identified independently of the patients who completed the patient survey; they were not the treating PCPs of these patients). There were large differences between patientreported concerns and the perception of patient concerns from the physician point of view, with physicians underestimating the importance of a number of patient-reported concerns. The largest percentage differences between the perceptions of patient survey respondents reported here and PCP survey respondents from the earlier study were observed for insulin ineffectiveness (patients' self-report as a concern, \% agree/ strongly agree: $40.9 \%$ vs physicians' perception of patients' concerns, $\%$ often/almost always/always $5.0 \%$ ), fear of mistakes during self-injection ( $52.6 \%$ vs $18.0 \%$ ), blood glucose monitoring (55.2\% vs $24.0 \%$ ), scarring/bruising from injection ( $35.7 \%$ vs $7.0 \%)$, and weight gain $(52.6 \%$ vs $23.0 \%)$. These may be areas where more proactive discussion, perhaps contrary to PCPs' perceptions, could lead to less concern and more buy-in from patients.

Physicians in our previous PCP survey ${ }^{23}$ perceived patients as highly adherent to treatment in the first three months postinitiation, and the majority also believed patients do not discontinue over 6 and 12 months. PCPs relied on clinical measures like fasting plasma glucose and $\mathrm{HbAlc}$, rather than direct conversations with patients to monitor their status and uncover underlying issues. In comparison, our previous claims analysis $^{9}$ indicated that $18.0 \%$ of new insulin initiators were classified as discontinuers over 1 year, and another $62.2 \%$ had an interruption. Previous studies using administrative claims have also found suboptimal persistence to basal insulin (with the percentage of persistent patients varying from 20 to 80 depending on the definition used). ${ }^{6,38-45}$ While any direct comparison between these claims-based findings and the PCP survey results of high adherence is limited by differences in methodology, the discrepancy does lead to more questions about how physicians become aware of and address interruptions in treatment.

While these studies have uncovered a range of information regarding both patient and physician behaviors, our findings also lead to additional questions such as the contribution of age differences to persistence behaviors and their interaction with other observed differences in patientreported behaviors and concerns. For example, older patients are more likely to be retired and able to maintain persistence given fewer work or family responsibilities. In addition, older patients are more likely to have had diabetes for a longer period of time, and may have tried more antidiabetic medications. Insulin may be their last treatment resort, and this could be an added motivator for continuing as there may be no or few other options beyond insulin.

Furthermore, a better understanding of interruptions in treatment, especially in the first 6 months after insulin initiation, is critical as such interruptions appear to have a direct bearing on patient outcomes. On the provider side, it would be useful to have in-depth knowledge of what 
steps physicians take to prevent interruptions, and how they influence patients to resume their insulin therapy. This will give insights into provider and health care system issues that may result in interruptions, which could help in formulating strategies to address them.

\section{Study Limitations}

This online survey study was subject to limitations common in survey research, such as generalizability, social desirability bias, and recall bias. Participants were recruited from the Harris Panel and third party panels in the US. Respondents who agree to participate in panels and complete an online survey could be different than the overall population of interest. Diagnosis of T2DM and insulin use could not be independently verified. Respondents may be more likely to select reasons for different patterns of persistence that they perceive to be more acceptable to others. For example, if respondents experienced side effects, they may be more likely to note side effects as a reason for interruption or discontinuation even though the primary reason might have been different. Such social desirability bias could have been mitigated by the confidentiality of survey responses, and the ability for respondents to select multiple factors or contributors to different persistence patterns. Multiple patient characteristics and self-reported behaviors and concerns may be correlated with persistence behaviors (such as age, concerns about weight gain, and treatment discontinuation), and additional research is warranted to describe correlation patterns and causalities.

\section{Conclusions}

Among adults with T2DM treated with insulin, there are frequent treatment interruptions and discontinuation. Gaps in insulin therapy place patients at risk for poor glycemic control, which could lead to long-term complications and increased medical costs. It is important to understand the reasons behind patients' persistence behaviors, and the differences in perceptions between healthcare providers and patients, as reconciliation of these perspectives is essential for successful T2DM management. Based on the results of our studies, patient buy-in when initiating insulin is likely to improve persistence, and earlier provider intervention, including explaining and addressing patients' concerns including potential side effects, may help reduce the amount of time patients are off their insulin therapy. Further opportunities on the part of providers to improve patient care around basal insulin initiation and maintenance include more frequent, direct outreach to patients to monitor adherence, preparation of insulin titration algorithms, and referrals to patient support groups.

\section{Ethics Approval and Consent to Participate}

This study was reviewed by the Western Institutional Review Board and exemption from review was granted. Informed consent was obtained for all participants. No patient-identifying information was collected.

\section{Acknowledgments}

Bernard Tulsi, MSc, Senior Medical Writer at HealthCore (at the time the manuscript was drafted) provided writing and editorial assistance for this manuscript.

\section{Author Contributions}

All authors contributed to data analysis, drafting and revising the article, gave final approval of the version to be published, and agree to be accountable for all aspects of the work.

\section{Funding}

Research funding was provided to HealthCore and Analysis Group by Eli Lilly and Company, in support of the Boehringer Ingelheim - Lilly Diabetes Alliance.

\section{Disclosure}

Kalirai was an employee of Eli Lilly and Company at the time the study was conducted. Perez-Nieves, Hadjiyianni, Pollom, and Reed are employees and stock holders of Eli Lilly and Company. Stephenson and Grabner are employees of HealthCore, Inc. Geremakis was an employee of HealthCore, Inc. at the time the study was conducted. Ivanova was an employee of Analysis Group, Inc. at the time the study was conducted. Fisher is a consultant to Eli Lilly and Company. The authors report no other conflicts of interest in this work.

\section{References}

1. Blak BT, Smith HT, Hards M, Curtis BH, Ivanyi T. Optimization of insulin therapy in patients with type 2 diabetes mellitus: beyond basal insulin. Diabet Med. 2012;29(7):e13-e20. doi:10.1111/j.14645491.2012.03586.x

2. Inzucchi SE, Bergenstal RM, Buse JB, et al. Management of hyperglycemia in type 2 diabetes: a patient-centered approach: position statement of the American Diabetes Association (ADA) and the European Association for the Study of Diabetes (EASD). Diabetes Care. 2012;35(6):1364-1379. doi:10.2337/dc12-0413

3. Sorli $\mathrm{C}$, Heile MK. Identifying and meeting the challenges of insulin therapy in type 2 diabetes. J Multidiscip Healthc. 2014;7:267-282. doi:10.2147/JMDH.S64084

4. Turner LW, Nartey D, Stafford RS, Singh S, Alexander GC. Ambulatory treatment of type 2 diabetes in the U.S., 1997-2012. Diabetes Care. 2014;37(4):985-992. doi:10.2337/dc13-2097 
5. Ishii H, Iwamoto $\mathrm{Y}$, Tajima N. An exploration of barriers to insulin initiation for physicians in Japan: findings from the Diabetes Attitudes, Wishes And Needs (DAWN) JAPAN study. PLoS One. 2012;7(6):e36361. doi:10.1371/journal.pone.0036361

6. IDF Diabetes Atlas - 7th Edition. International Diabetes Federation. Available from: http://www.diabetesatlas.org/. Accessed May 11, 2016.

7. Inzucchi SE, Bergenstal RM, Buse JB, et al. Management of hyperglycemia in type 2 diabetes, 2015: a patient-centered approach: update to a position statement of the American Diabetes Association and the European Association for the Study of Diabetes. Diabetes Care. 2015;38(1):140-149. doi:10.2337/dc14-2441

8. Jones S, Benroubi M, Castell C, et al. Characteristics of patients with type 2 diabetes mellitus initiating insulin therapy: baseline data from the INSTIGATE study. Curr Med Res Opin. 2009;25(3):691-700. doi:10.1185/03007990902739669

9. Perez-Nieves M, Kabul S, Desai U, et al. Basal insulin persistence, associated factors, and outcomes after treatment initiation among people with type 2 diabetes mellitus in the US. Curr Med Res Opin. 2016;32(4):669-680. doi:10.1185/03007995.2015.1135789

10. Watson L, Das R, Farquhar R, Langerman H, Barnett AH. Consequences of delaying treatment intensification in type 2 diabetes: evidence from a UK database. Curr Med Res Opin. 2016;32 (9):1465-1475. doi:10.1185/03007995.2016.1157462

11. Zografou I, Strachan M, McKnight J. Delay in starting insulin after failure of other treatments in patients with type 2 diabetes mellitus. Hippokratia. 2014;18(4):306-309.

12. Brown MT, Bussell JK. Medication adherence: WHO cares? Mayo Clin Proc. 2011;86(4):304-314. doi:10.4065/mcp.2010.0575

13. Davies MJ, Gagliardino JJ, Gray LJ, Khunti K, Mohan V, Hughes R. Real-world factors affecting adherence to insulin therapy in patients with Type 1 or Type 2 diabetes mellitus: a systematic review. Diabet Med. 2013;30(5):512-524. doi:10.1111/dme.2013.30.issue-5

14. Polonsky WH, Henry RR. Poor medication adherence in type 2 diabetes: recognizing the scope of the problem and its key contributors. Patient Prefer Adherence. 2016;10:1299-1307. doi:10.2147/PPA

15. Rozenfeld Y, Hunt JS, Plauschinat C, Wong KS. Oral antidiabetic medication adherence and glycemic control in managed care. $\mathrm{Am}$ J Manag Care. 2008;14(2):71-75.

16. Simpson SH, Eurich DT, Majumdar SR, et al. A meta-analysis of the association between adherence to drug therapy and mortality. $B M J$. 2006;333(7557):15. doi:10.1136/bmj.38875.675486.55

17. Garcia-Perez LE, Alvarez M, Dilla T, Gil-Guillen V, Orozco-Beltran D. Adherence to therapies in patients with type 2 diabetes. Diabetes Ther. 2013;4(2):175-194. doi:10.1007/s13300-013-0034-y

18. Peyrot M, Barnett AH, Meneghini LF, Schumm-Draeger PM. Insulin adherence behaviours and barriers in the multinational global attitudes of patients and physicians in insulin therapy study. Diabet Med. 2012;29(5):682-689. doi:10.1111/j.14645491.2012.03605.x

19. Peyrot M, Rubin RR, Khunti K. Addressing barriers to initiation of insulin in patients with type 2 diabetes. Prim Care Diabetes. 2010;4 (Suppl 1):S11-S18. doi:10.1016/S1751-9918(10)60004-6

20. Polonsky WH, Fisher L, Guzman S, Villa-Caballero L, Edelman SV. Psychological insulin resistance in patients with type 2 diabetes: the scope of the problem. Diabetes Care. 2005;28(10):2543-2545. doi:10.2337/diacare.28.10.2543

21. Polonsky WH, Hajos TR, Dain MP, Snoek FJ. Are patients with type 2 diabetes reluctant to start insulin therapy? An examination of the scope and underpinnings of psychological insulin resistance in a large, international population. Curr Med Res Opin. 2011;27 (6):1169-1174. doi:10.1185/03007995.2011.573623

22. Oliveria SA, Menditto LA, Ulcickas Yood M, Koo YH, Wells KE, McCarthy BD. Barriers to the initiation of, and persistence with, insulin therapy. Curr Med Res Opin. 2007;23(12):3105-3112. doi: $10.1185 / 030079907 X 242638$
23. Kalirai S, Stephenson J, Perez-Nieves M, et al. Primary care physician perspectives on basal insulin initiation and maintenance in patients with type 2 diabetes mellitus. Prim Care Diabetes. 2017.

24. Perez-Nieves M, Ivanova JI, Hadjiyianni I, et al. Basal insulin initiation use and experience among people with type 2 diabetes mellitus with different patterns of persistence: results from a multi-national survey. Curr Med Res Opin. 2017;33:1-10.

25. Peyrot M, Perez-Nieves M, Ivanova J, et al. Correlates of basal insulin persistence among insulin-naive people with type 2 diabetes: results from a multinational survey. Curr Med Res Opin. 2017;33 (10):1843-1851. doi:10.1080/03007995.2017.1341868

26. American Diabetes Association. Standards of medical care in diabetes-2017: summary of Revisions. Diabetes Care. 2017;40 (Suppl 1):S4-S5. doi:10.2337/dc17-S003

27. Type 2 diabetes: international survey shows quality of patient-physician conversation at diagnosis linked to better patient outcomes. Available from: https:/www.boehringer-ingelheim.com/ press-release/type-2-diabetes-international-survey-shows-qualitypatient-physician-conversation. Accessed May 11, 2016.

28. Anderson RM, Funnell MM. Patient empowerment: reflections on the challenge of fostering the adoption of a new paradigm. Patient Educ Couns. 2005;57(2):153-157. doi:10.1016/j.pec.2004.05.008

29. Atreja A, Bellam N, Levy SR. Strategies to enhance patient adherence: making it simple. MedGenMed. 2005;7(1):4.

30. Carman KL, Dardess P, Maurer M, et al. Patient and family engagement: a framework for understanding the elements and developing interventions and policies. Health Aff (Millwood). 2013;32(2):223-231. doi:10.1377/ hlthaff.2012.1133

31. LaSalle JR, Berria R. Insulin therapy in type 2 diabetes mellitus: a practical approach for primary care physicians and other health care professionals. J Am Osteopath Assoc. 2013;113(2):152-162.

32. Nagelkerk J, Reick K, Meengs L. Perceived barriers and effective strategies to diabetes self-management. $J$ Adv Nurs. 2006;54 (2):151-158. doi:10.1111/j.1365-2648.2006.03799.x

33. Rendle KA, May SG, Uy V, Tietbohl CK, Mangione CM, Frosch DL. Persistent barriers and strategic practices: why (asking about) the everyday matters in diabetes care. Diabetes Educ. 2013;39 (4):560-567. doi:10.1177/0145721713492218

34. Schwartz DD, Stewart SD, Aikens JE, Bussell JK, Osborn CY, Safford MM. Seeing the person, not the illness: promoting diabetes medication adherence through patient-centered collaboration. Clin Diabetes. 2017;35(1):35-42. doi:10.2337/cd16-0007

35. Funnell MM, Brown TL, Childs BP, et al. National standards for diabetes self-management education. Diabetes Care. 2008;31(Suppl 1):S97-S104. doi:10.2337/dc08-S097

36. Funnell MM, Kruger DF, Spencer M. Self-management support for insulin therapy in type 2 diabetes. Diabetes Educ. 2004;30 (2):274-280. doi:10.1177/014572170403000220

37. Haas L, Maryniuk M, Beck J, et al. National standards for diabetes self-management education and support. Diabetes Care. 2012;35 (11):2393-2401. doi:10.2337/dc12-1707

38. Baser O, Tangirala K, Wei W, Xie L. Real-world outcomes of initiating insulin glargine-based treatment versus premixed analog insulins among US patients with type 2 diabetes failing oral antidiabetic drugs. Clinicoecon Outcomes Res. 2013;5:497-505. doi: $10.2147 /$ CEOR

39. Bonafede MM, Kalsekar A, Pawaskar M, et al. A retrospective database analysis of insulin use patterns in insulin-naive patients with type 2 diabetes initiating basal insulin or mixtures. Patient Prefer Adherence. 2010;4:147-156. doi:10.2147/PPA.S10467

40. Cooke CE, Lee HY, Tong YP, Haines ST. Persistence with injectable antidiabetic agents in members with type 2 diabetes in a commercial managed care organization. Curr Med Res Opin. 2010;26(1):23 1-238. doi:10.1185/03007990903421994 
41. Davis KL, Tangirala M, Meyers JL, Wei W. Real-world comparative outcomes of US type 2 diabetes patients initiating analog basal insulin therapy. Curr Med Res Opin. 2013;29(9):1083-1091. doi:10.1185/03007995.2013.811403

42. Thayer S, Wei W, Buysman E, et al. The INITIATOR study: pilot data on real-world clinical and economic outcomes in US patients with type 2 diabetes initiating injectable therapy. Adv Ther. 2013;30 (12):1128-1140. doi:10.1007/s12325-013-0074-8

43. Wang L, Wei W, Miao R, Xie L, Baser O. Real-world outcomes of US employees with type 2 diabetes mellitus treated with insulin glargine or neutral protamine Hagedorn insulin: a comparative retrospective database study. BMJ Open. 2013;3(4):e002348. doi:10.1136/ bmjopen-2012-002348
44. Wei W, Pan C, Xie L, Baser O. Real-world insulin treatment persistence among patients with type 2 diabetes. Endocr Pract. 2014;20 (1):52-61. doi:10.4158/EP13159.OR

45. Ascher-Svanum H, Lage MJ, Perez-Nieves M, et al. Early discontinuation and restart of insulin in the treatment of type 2 diabetes mellitus. Diabetes Ther. 2014;5(1):225-242. doi:10.1007/s13300-01 4-0065-z

Diabetes, Metabolic Syndrome and Obesity: Targets and Therapy

\section{Publish your work in this journal}

Diabetes, Metabolic Syndrome and Obesity: Targets and Therapy is an international, peer-reviewed open-access journal committed to the rapid publication of the latest laboratory and clinical findings in the fields of diabetes, metabolic syndrome and obesity research. Original research, review, case reports, hypothesis formation, expert opinion and commentaries are all considered for publication. The manuscript management system is completely online and includes a very quick and fair peer-review system, which is all easy to use. Visit http://www.dovepress.com/testimonials.php to read real quotes from published authors. 